\title{
Aplicação da TRI na versão brasileira do Defensive Style Questionnaire (DSQ)
}

\author{
Application of IRT in the Brazilian version of the Defensive Style Questionnaire (DSQ)
}

\author{
Lucas de Francisco Carvalho ${ }^{[a]}$, Ricardo Primimib], Fernando Pessotto ${ }^{[c]}$
}

\footnotetext{
${ }^{[a]}$ Doutor em Psicologia pela Universidade São Francisco, docente do programa de pósgraduação stricto sensu em Psicologia da Universidade São Francisco, Itatiba, São Paulo, Brasil, e-mail: lucas@labape.com.br

${ }^{[b]}$ Doutor em Psicologia escolar e do desenvolvimento pela Universidade de São Paulo, docente do programa de pós-graduação stricto sensu em Psicologia da Universidade São Francisco, Itatiba, São Paulo, Brasil, email: rprimi@mac.com

${ }^{[c]}$ Mestre e doutorando em Psicologia pela Universidade São Francisco, docente do curso de graduação em Psicologia da UNISAL, Americana, São Paulo, Brasil; email: fpessotto@yahoo.com.br
}

Recebido: 27/02/2013 Received: 02/27/2013

\section{Resumo}

Atualmente, alguns autores entendem que os mecanismos de defesa são organizados em um continuum que varia de imaturo a defesas mais maduras. Sob essa perspectiva, foi desenvolvido o Defensive Style Questionnaire 40 (DSQ-40), sendo um instrumento de autorrelato composto por 40 itens que devem ser respondidos em uma escala Likert de sete pontos, distribuídos em três fatores (maduro, neurótico e imaturo). O DSQ-40 foi traduzido e adaptado para o Brasil e teve suas propriedades psicométricas verificadas por meio da Teoria Clássica dos Testes (TCT). O presente estudo teve como objetivo verificar os parâmetros dos itens e pessoas obtidos por meio do modelo de escala graduada para o DSQ-40. Para tanto, foram recrutados 105 participantes com idade entre 19 e 49 anos $(M=23,64$; $D P=5,14)$, sendo 59 mulheres. As análises realizadas sustentam a unidimensionalidade dos fatores do instrumento, sugerem a adequação de uma escala Likert de três pontos para o instrumento, confirmam que os mecanismos de defesa mais adaptativos são mais comuns na população e demonstram índices de fidedignidade satisfatórios de acordo com os níveis de theta dos sujeitos.

Palavras-chave: Teoria de Resposta ao Item. Avaliação psicológica. Psicometria.

\section{Abstract}

Currently, some authors believe that the defense mechanisms are organized on a continuum that ranges from immature to more mature defenses. From this perspective, the Defensive Style Questionnaire 40 (DSQ-40) was developed, being a self-report instrument consisting of 40 items that must be answered on aseven s Likert scale of seven points, distributed among three factors (mature, neurotic and immature). The DSQ-40 was translated and adapted for Brazil and had its psychometric properties verified by Classical Test Theory (CTT). The present study aimed to determine the parameters of the items and people obtained through the rating scale model for the DSQ-40. Thereunto, we recruited 105 participants aged between 19 and 49 years $(M=23.64, S D=5.14)$, with 59 women. The analyses support the unidimensionality of the instrument factors, suggest the appropriateness of athree s Likert scale of three points for the instrument, confirm that the most adaptive defense mechanisms are more common in the population and demonstrate good reliability in accordance with the levels of theta subjects.

Keywords: Item Response Theory. Psychological assessment. Psychometrics. 


\section{Introdução}

Os mecanismos de defesa foram descritos primeiramente por Sigmund Freud (1976) como uma forma de disfarce do ego utilizada pelos indivíduos. Anna Freud (1937) também estudou o tema acrescentando que, apresentando patologias ou não, cada pessoa possui um repertório desses mecanismos, sendo eles processos mentais utilizados pelo ego contra as demandas instintivas do id, utilizados na regulação com os conflitos.

Em uma perspectiva contemporânea, Blaya et al. (2006) complementam que os mecanismos de defesa são organizados em um continuum que varia de imaturo a defesas maduras, sendo uma estrutura individual importante da personalidade. Algumas defesas imaturas estão relacionadas a diagnósticos psiquiátricos como depressão e pânico, por exemplo, envolvendo situações em que o indivíduo não consegue lidar com os elementos estressores internos ou externos. A manifestação de defesas mais ou menos maduras varia de indivíduo para indivíduo, como propõem Zaslavsky e Escobar (2003).

Considerando o continuum (Blaya et al., 2006), e não contraditório ao entendimento inicial de Freud, de acordo com o DSM-IV-TR-TR (APA, 2002) os mecanismos de defesa são "processos psicológicos automáticos que protegem o indivíduo da ansiedade e da consciência de estressores ou perigos internos ou externos" (p, 754). Portanto, os mecanismos de defesa são responsáveis pela intermediação dos comportamentos de um indivíduo frente a conflitos, podendo ser adaptativos ou não, dependendo de sua gravidade, inflexibilidade e contexto em que ocorrem. Especificamente acerca da adaptação desses mecanismos, o DSM-IV-TR-TR apresenta uma classificação composta por sete níveis de defesa de acordo com as características individuais e tipo de enfrentamento de conflito, descritos na sequência. Vale ressaltar que os mecanismos estão descritos dos mais adaptativos aos menos adaptativos, com ordem numérica crescente, conforme apresentados na Tabela 1, e também estão apresentados os mecanismos específicos em cada um dos níveis. Especificamente na coluna "breve definição", estão apontados os modelos com o que o indivíduo lida com o conflito.

No alto nível adaptativo (1) o sujeito apresenta um manejo bastante favorável em relação ao conflito, maximizando a gratificação e permitindo a conscientização de sentimentos, ideias e suas consequências. Alguns exemplos são afiliação, humor, altruísmo entre outros. 0 segundo nível (2) é o das inibições mentais, em que se mantêm ideias ou sentimentos ameaçadores fora da consciência, como é o caso da anulação, dissociação e repressão. Já o nível de leve distorção da imagem (3), consiste na distorção da imagem de si mesmo ou dos outros, como a desvalorização, idealização e onipotência. No nível de negação (4) o sujeito exclui da consciência o que é ruim, estabelecendo uma atribuição, coerente ou não, das causas externas, como na negação, projeção e racionalização. 0 nível de importante distorção de imagem (5) é caracterizado por uma ampla distorção ou descrição incorreta da própria imagem ou do outro, sendo alguns exemplos a cisão da autoimagem ou da imagem alheia, fantasia autista e identificação projetiva. No nível de ação (6) o sujeito apresenta um funcionamento defensivo caracterizado pela ação ou retraimento contra os estressores, sendo alguns exemplos a atuação, a agressividade passiva, retraimento, entre outros. Por fim, o nível de desregulação defensiva (7) é caracterizado pelo fracasso na regulação em conter os elementos estressores, como é o caso de quadros como distorção ou negação psicótica, projeção delirante entre outros.

Sendo os mecanismos de defesa manifestações não mensuráveis diretamente, sua avaliação é feita por intermédio de ações conscientes que parecem estar diretamente ligadas a esses fenômenos (Kipper et al., 2004). Um dos instrumentos desenvolvidos para esta mensuração é o Defensive Style Questionnaire ([DSQ-40]; Andrews, Sing \& Bond, 1993), elaborado para avaliar derivados conscientes dos mecanismos de defesa baseado nos comportamentos característicos ao lidar com conflitos, concebendo serem capazes de refletir sobre como enfrentam situação conflitivas. Tratase de um instrumento de autorrelato composto por 40 itens que devem ser respondidos por meio de uma escala Likert de nove pontos (variando entre "discordo completamente" e "concordo plenamente"). Os itens do DSQ-40 estão organizados em três fatores, sendo eles: imaturo (projeção, agressão passiva, acting out, isolamento, desvalorização, fantasia autística, negação, deslocamento, dissociação, cisão e somatização), maduro (sublimação, humor, antecipação, supressão e racionalização correspondem ao primeiro fator) e 
Tabela 1 - Mecanismos de defesa de acordo com o DSM-IV-TR.

\begin{tabular}{|c|c|c|}
\hline Nível adaptativo & Mecanismos & Breve definição \\
\hline \multirow{8}{*}{1} & Afiliação & Busca a ajuda de outros. \\
\hline & Altruísmo & Por meio da satisfação da necessidade alheia. \\
\hline & Antecipação & Antecipa reações e/ou futuro. \\
\hline & Autoafirmação & Expressa-se verbal e emocionalmente. \\
\hline & Auto-observação & Reflete sobre seus próprios sentimentos e pensamentos. \\
\hline & Humor & Salienta os aspectos divertidos ou irônicos. \\
\hline & Sublimação & Canaliza os impulsos mal adaptativos para comportamentos aceitáveis. \\
\hline & Supressão & Evita pensar em problemas e desejos perturbadores. \\
\hline \multirow{7}{*}{2} & Anulação & $\begin{array}{l}\text { Utiliza comportamentos para negar ou corrigir simbolicamente pensamentos e } \\
\text { ações inaceitáveis. }\end{array}$ \\
\hline & Deslocamento & Transfere sentimentos e respostas de um objeto para outro. \\
\hline & Dissociação & Há ruptura em funções habitualmente integradas. \\
\hline & Formação reativa & Substitui elementos internos inaceitáveis por elementos diretamente opostos. \\
\hline & Intelectualização & Uso excessivo do pensamento abstrato ou de generalizações. \\
\hline & Isolamento do afeto & Separação entre as ideias e sentimentos associados. \\
\hline & Repressão & Expele desejos, pensamentos e experiências da consciência. \\
\hline \multirow{3}{*}{3} & Desvalorização & Atribui qualidades negativas a si e outros. \\
\hline & Idealização & Atribui a outros qualidades positivas exageradas. \\
\hline & Onipotência & Considera-se detentor de capacidades especiais, superior aos outros. \\
\hline \multirow{3}{*}{4} & Negação & Recusa-se a reconhecer aspectos dolorosos da realidade. \\
\hline & Projeção & Atribui os próprios sentimentos, impulsos e pensamentos a outras pessoas. \\
\hline & Racionalização & Encobre suas verdadeiras motivações por meio de explicações. \\
\hline \multirow{3}{*}{5} & Cisão & $\begin{array}{l}\text { Compartimenta estados afetivos opostos, não sendo capaz de integrar } \\
\text { qualidades positivas e negativas. }\end{array}$ \\
\hline & Fantasia autista & Utiliza devaneios excessivos. \\
\hline & Identificação projetiva & $\begin{array}{l}\text { Atribui seus impulsos inaceitáveis a outras pessoas, contudo, permanece cons- } \\
\text { ciente de seus impulsos. }\end{array}$ \\
\hline \multirow{4}{*}{6} & Autação (acting out) & Utiliza a ação no lugar da reflexão. \\
\hline & Agressividade passiva & Expressa agressão indireta e não declaradamente. \\
\hline & Queixa com rejeição & Queixas repetidas sem aceitar ajuda alheia. \\
\hline & Retraimento apático & -- \\
\hline \multirow{3}{*}{7} & Distorção psicótica & -- \\
\hline & Negação psicótica & -- \\
\hline & Projeção delirante & -- \\
\hline
\end{tabular}

Nota. Os mecanismos e definições apresentadas foram retirados do DSM-IV-TR (APA, 2002). Contudo, para alguns mecanismos não foram apresentadas definições, como é o caso de todos os mecanismos do nível (7) da desregulação defensiva.

neurótico (anulação, pseudo-altruísmo, idealização e formação reativa) totalizando 20 mecanismos de defesa.

No Brasil foram realizadas duas traduções e adaptações culturais do DSQ-40, a primeira por Blaya et al. (2004) e a segunda por Andrade e Shirakawa (2006). Neste estudo, o foco é dado para a versão de Blaya et al. (2004), já que essa versão foi utilizada na coleta de dados.

Concernente aos procedimentos de tradução e adaptação do DSQ-40 (Blaya et al., 2004), duas versões em português foram elaboradas, uma por um psiquiatra bilíngue e outra por um tradutor. As versões foram comparadas e compiladas numa 
versão preliminar do instrumento. Na sequência, realizou-se uma análise de conteúdo solicitando aos especialistas que correlacionassem cada item com um mecanismo de defesa conforme descrito no DSM-IV-TR, quando disponível. Após esse processo, algumas alterações foram realizadas no que se refere a termos e adaptações linguísticas. Finalmente, o material foi retraduzido para o inglês e enviado para o autor original, que corroborou com a versão, estabelecendo-se assim a versão brasileira final do instrumento.

No que se refere às propriedades psicométricas da versão brasileira do DSQ-40, Blaya et al. (2007) realizaram um estudo buscando por evidências de validade com base no construto, contando com 339 sujeitos. A amostra foi composta por 66 sujeitos em psicoterapia com idade média de 35,3 anos ( $\mathrm{DP}=10,9), 237$ pacientes psiquiátricos com diferentes diagnósticos, sendo eles depressão $(\mathrm{N}=40$; idade $M=49,1 ; D P=11,5)$, fobia $(N=35$; idade $M=33,1$; $\mathrm{DP}=12$,2), pânico ( $\mathrm{N}=129$; idade $\mathrm{M}=38,1 ; \mathrm{DP}=10,3)$, transtorno obsessivo-compulsivo $(\mathrm{N}=33$; idade $\mathrm{M}=35$,3; $\mathrm{DP}=10,9$ ) e um grupo controle com 36 funcionários de um hospital sem histórico de diagnóstico psiquiátrico, aos quais os dados sociodemográficos não foram indicados. Foram utilizados, ainda, o MMPI e a Escala de Funcionamento Defensivo (DFS). A análise fatorial exploratória apontou para uma solução de três fatores, correspondentes aos fatores da versão original do instrumento, explicando $38,1 \%$ da variância da amostra. Verificou-se também que somente para a classificação imaturo (fator I), a consistência interna demonstrou-se adequada com um $\alpha$ de Cronbach de 0,77, enquanto para maduro (fator II) e neurótico (fator III) foram respectivamente de 0,55 e 0,52. A fidedignidade também foi verificada por meio do teste-reteste para o grupo controle, em um intervalo de quatro meses, não sendo encontradas diferenças significativas entre as aplicações e com magnitudes de correlação que variaram entre 0,68 e 0,81 .

Buscando agregar evidências de validade para o DSQ-40, Galvão (2007) realizou um estudo a partir de uma amostra composta por 271 internos de penitenciárias com idade variando entre 19 e 69 anos $(\mathrm{M}=33,06$ e $\mathrm{DP}=10.97)$, sendo 63 mulheres. Parte da amostra respondeu à Escala de Resiliência, sendo verificada uma correlação moderada $(r=0,58$; $p=0,001$ ) entre as escalas. A análise fatorial indicou um coeficiente KMO de 0,82 , sinalizando covariância suficientemente adequada ao instrumento. Quanto à precisão o índice obtido no teste-reteste apontou baixa estabilidade temporal das medidas $(r=0,611$; $p<0,05$ ).

Os estudos nacionais e internacionais para verificação das propriedades psicométricas do DSQ-40, encontrados para o desenvolvimento desta pesquisa, apontam para a adequação dessa ferramenta. Especificamente, a versão nacional demonstrou estrutura interna similar à encontrada para a versão original do instrumento. Este estudo insere-se na continuidade de buscas de evidências de validade para a versão brasileira do DSQ-40. Para tanto, serão utilizados análises de dados com base na Teoria de Resposta ao Item (TRI).

0 uso dos modelos matemáticos com base na TRI permite: (a) investigar a estrutura e adequação das categorias utilizadas como respostas de um teste (sobretudo no caso de escalas tipo Likert); (b) comparar o nível de intensidade do construto representado nos itens de um teste com o nível de intensidade do construto nas pessoas; (c) investigar a organização hierárquica dos itens de acordo com a intensidade representada por cada um deles; e, (d) verificar os índices de fidedignidade de um teste nos diferentes níveis em que o construto é mensurado. Outras vantagens e possibilidades de aplicação da TRI poderiam ser apontadas, mas um levantamento extenso vai além do escopo deste trabalho.

A TRI propõe um modelo matemático para representar a situação de testagem na qual uma pessoa responde a um grupo de itens. Quanto mais intensa é uma determinada característica na pessoa, maior é a probabilidade de endosso com um item que avalie aquela característica. Diferentemente, quanto menos intensa é a característica na pessoa, menor deve ser a probabilidade de endossar o item (Pasquali \& Primi, 2007). Portanto, a probabilidade de escolha a uma resposta em particular varia de acordo com o nível em que uma dada característica ( $\theta$ ou theta) é presente ou não no respondente.

Existem diversos modelos baseados na TRI, sendo um dos mais utilizados o modelo de Rasch (Embretson \& Reise, 2000). No modelo de Rasch, os itens são caracterizados somente pelo parâmetro b, chamado de nível de dificuldade, por isso esse modelo também é chamado de modelo de um parâmetro. Para o presente estudo, considerando que o DSQ-40 é composto por itens que devem ser respondidos em escala Likert, utilizou-se o 
modelo de escala graduada (Rating Scale Model), que é uma das propostas dentro da família de modelos de Rasch.

Considerando a possibilidade de uso desse modelo matemático no campo de avaliação dos mecanismos de defesa, o objetivo do presente estudo foi verificar os parâmetros dos itens e pessoas obtidos por meio do modelo de escala graduada para o DSQ40. Especificamente, investigou-se a dimensionalidade dos fatores do instrumento; foram estimados os parâmetros dos itens do teste e participantes do estudo; verificou-se o ajuste dos parâmetros encontrados ao que é esperado pelo modelo matemático; os índices de fidedignidade e a precisão local foram estimados; buscou-se analisar as categorias de resposta das escalas; e, procedeu-se a análises do mapa de itens. A explanação dos procedimentos empregados será apresentada convenientemente ao longo do trabalho.

\section{Método}

\section{Participantes}

Foram recrutados 105 participantes de uma cidade do interior do estado de São Paulo, com idade variando entre 19 e 49 anos ( $M=23,64 ; \mathrm{DP}=5,14)$, sendo 45 homens $(42,9 \%), 59$ mulheres $(56,2 \%)$, e $1(1,0 \%)$ caso omisso. Todos os participantes tinham curso superior incompleto, sendo 42 (40\%) do curso de psicologia, $34(32,4 \%)$ da engenharia, $6(5,7 \%)$ da administração e $23(21,9 \%)$ casos omissos.

\section{Instrumentos}

O DSQ-40 é um instrumento de autorrelato, composto por 40 itens, que foi traduzido e adaptado para o Brasil por Blaya et al. (2004). Esse instrumento tem o objetivo de avaliar os derivativos conscientes de 20 mecanismos de defesa, divididos em três fatores: maduro, neurótico e imaturo. O DSQ-40 deve ser respondido a partir de uma escala Likert de 9 pontos, em que 1 indica "discordo completamente" e 9 indica "concordo plenamente". 0 tempo estimado para aplicação desse instrumento é de 10 minutos.

\section{Procedimento e Delineamento}

O instrumento foi aplicado nos participantes, sendo que para todos foi entregue o Termo de Consentimento Livre e Esclarecido (TCLE) no qual constou o objetivo principal do estudo e a divulgação dos resultados de acordo com as normas éticas (Protocolo CAAE 0350.0.142.000-08). Assim, somente concordando com os procedimentos da pesquisa e assinando o TCLE o participante foi habilitado a participar deste estudo. Ao lado disso, o momento da aplicação dos instrumentos implicou sempre a presença de um pesquisador, possibilitando que possíveis dúvidas dos participantes fossem esclarecidas. Apesar disso, raríssimas foram as ocasiões nas quais surgiram dúvidas.

As aplicações foram realizadas em salas de aula de uma universidade particular do interior do estado de São Paulo. Após a coleta de dados e a tabulação dos mesmos, foram realizadas análises estatísticas buscando responder o objetivo do estudo. Os dados coletados foram submetidos à análise por meio do modelo de Rasch, especificamente o modelo de resposta gradual, utilizando o software estatístico Winsteps (Linacre, 2009) verificando os parâmetros dos itens e dos respondentes.

Cabe considerar que um dos postulados básicos da modelagem via TRI é a unidimensionalidade, isto é, o modelo supõe que os itens estejam medindo uma dimensão principal e que dimensões secundárias tenham uma influência negligenciável (Hambleton \& Swaminatham, 1985). Assim, a verificação da unidimensionalidade das dimensões do DSQ-40 foi o passo inicial para proceder-se às outras análises apresentadas neste estudo. Para tanto, considerou-se cada fator do DSQ-40 como uma dimensão independente.

Empregou-se o Winsteps, para calibrar os parâmetros dos itens (características), que implementa um método de estimação de máxima verossimilhança (Joint Maximum Likelihood Estimation). Para análise do ajuste do modelo, foram considerados os índices de ajuste ao modelo, infit e outfit. Esses índices consistem em valores médios dos resíduos (pontuação observada - modelada) padronizados e elevados ao quadrado, sendo assim, são qui-quadrados divididos pelos graus de liberdade. Empregando as recomendações da literatura, considerou-se valores acima de 1,3 e correlações item-total próximas a zero como indicativos de desajuste ao modelo 
(Smith, 1996; Wright \& Linacre, 1994). Também foram calculados índices de fidedignidade e precisão local; categorias de respostas das escalas; e análises quantitativas e qualitativas do mapa de itens. Cabe ressaltar, também, que para fins das análises, a média de dificuldade dos itens (b) foi fixada em zero.

\section{Resultados e Discussão}

Visando a verificação do pressuposto da unidimensionalidade para o DSQ-40, foi utilizada a análise de componentes principais dos resíduos implementada no Winsteps. A partir dos parâmetros dos itens e dos sujeitos, é possível calcular uma resposta esperada para cada sujeito a cada item. A discrepância entre essa resposta modelada (esperada) e a observada é considerada como resíduo.

A análise de componentes principais que o Winsteps procede é realizada sobre essa nova matriz de dados de resíduos, isto é, com base na parcela de respostas não preditas pelo modelo. Assim, se um componente reunir itens com magnitude maior que 2 (Linacre, 2009), sugere-se uma segunda dimensão que potencialmente pode afetar os dados de maneira a ofuscar o significado da primeira dimensão. Isso posto, nessa análise busca-se verificar componentes com valores de eigenvalues iguais ou superiores a 2,0 .

Verificou-se a possibilidade de unidimensionalidade no conjunto total de itens do instrumento; contudo, foram encontrados resíduos superiores a 2,0. Assim, procedeu-se a análise considerando separadamente cada um dos fatores do instrumento. A partir dessa análise, observou-se que apenas o fator três apresentou resíduo superior a 2,0 eigenvalues.

Considerando que o fator três apresentou um primeiro contraste com eigenvalue igual a 2,3, foi utilizado o procedimento indicado por Linacre (2009). A partir desse procedimento, os thetas dos sujeitos foram salvos com base no grupo de itens no polo positivo do contraste $(29,11,36,14$, $12,27,33,37,31,19,23$ e 13 ) e o mesmo foi feito para o polo negativo $(15,18,9,6,22,10,8,20,34$ e 17). Os thetas salvos com base em cada um dos grupos de itens foram exportados para o software Statistical Package for the Social Sciences (SPSS) e correlacionados. 0 pressuposto subjacente a esse procedimento é que ambos os grupos de thetas gerados estejam relacionados prioritariamente a um mesmo construto. Ainda, a magnitude da correlação de Pearson, utilizada para verificar o pressuposto subjacente, foi corrigida pelo procedimento de desatenuação de erro $(\mathrm{r} / \sqrt{ }(\alpha 1 \times \alpha 2)$; onde $r$ é a magnitude de correlação e $\alpha$ é o índice de consistência interna). A partir disso, a magnitude encontrada entre os dois conjuntos de itens foi igual a 0,45 . Não foi encontrado na literatura um coeficiente mínimo aceitável sugerindo unidimensionalidade entre os grupos de itens. Tendo em vista que a magnitude de correlação encontrada é compreendida tradicionalmente como moderada, mas pode também ser entendida como alta (Hemphill, 2003), neste estudo a magnitude encontrada foi considerada como suficiente para proceder às análises com o fator três.

Uma vez garantida a unidimensionalidade de cada fator, deu-se prosseguimento às análises investigando-se o funcionamento das categorias de resposta dos fatores do DSQ-40. A análise das categorias de resposta permite observar se as categorias na escala Likert proposta para o instrumento obtiveram um funcionamento minimamente adequado (Figura 1).

Na Figura 1 estão demonstradas as categorias de resposta dos itens dos três fatores do DSQ-40. No eixo x observa-se a escala de theta (nível dos respondentes no traço latente) e no eixo y a probabilidade de resposta dos participantes nos diferentes níveis de theta (na figura, a média de b está centrada em zero). Desse modo, observam-se as probabilidades de endosso dos participantes em cada uma das categorias de resposta e suas distribuições nos diferentes níveis de theta para um item $b_{i}=0$ (isto é, nível médio de dificuldade igual à zero). A intersecção entre duas categorias pode ser interpretada como o valor limiar (threshold) de transição entre essas categorias. Verifica-se que em todos os fatores houve sobreposição das curvas, de modo que algumas categorias de resposta não apresentaram alguma região na escala de theta (eixo horizontal) na qual seria a mais provável. Por isso, por meio do procedimento collapsed no programa Winsteps, as categorias de resposta foram agregadas entre si. Buscou-se agregar as categorias de modo que as mantidas apresentassem uma faixa clara onde seria a mais provável na escala de theta e que aumentasse a fidedignidade do conjunto de itens. A Figura 2 apresenta as categorias de resposta após o procedimento para coalisão entre elas. 

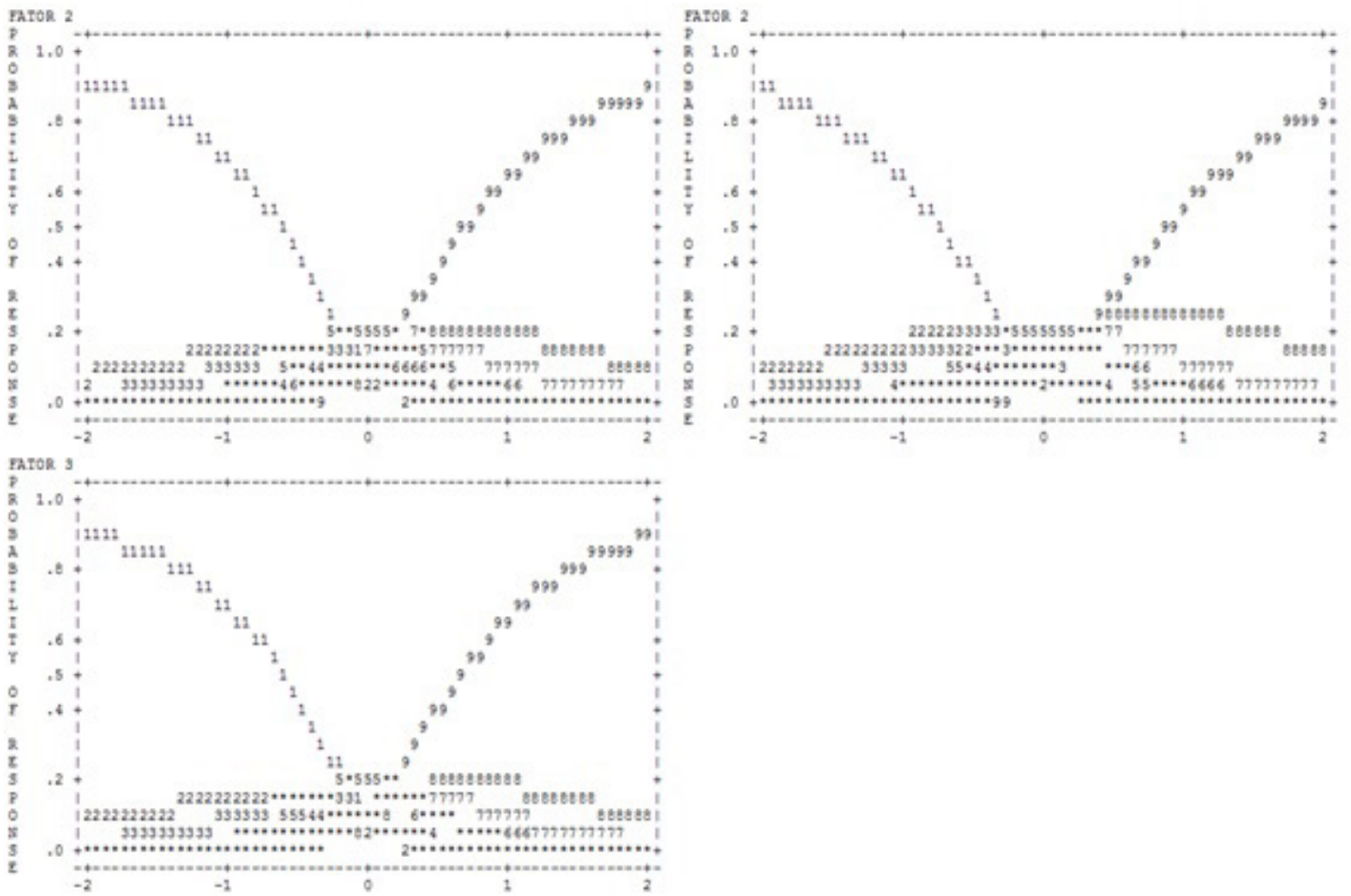

Figura 1 - Categorias de resposta dos fatores do DSQ-40
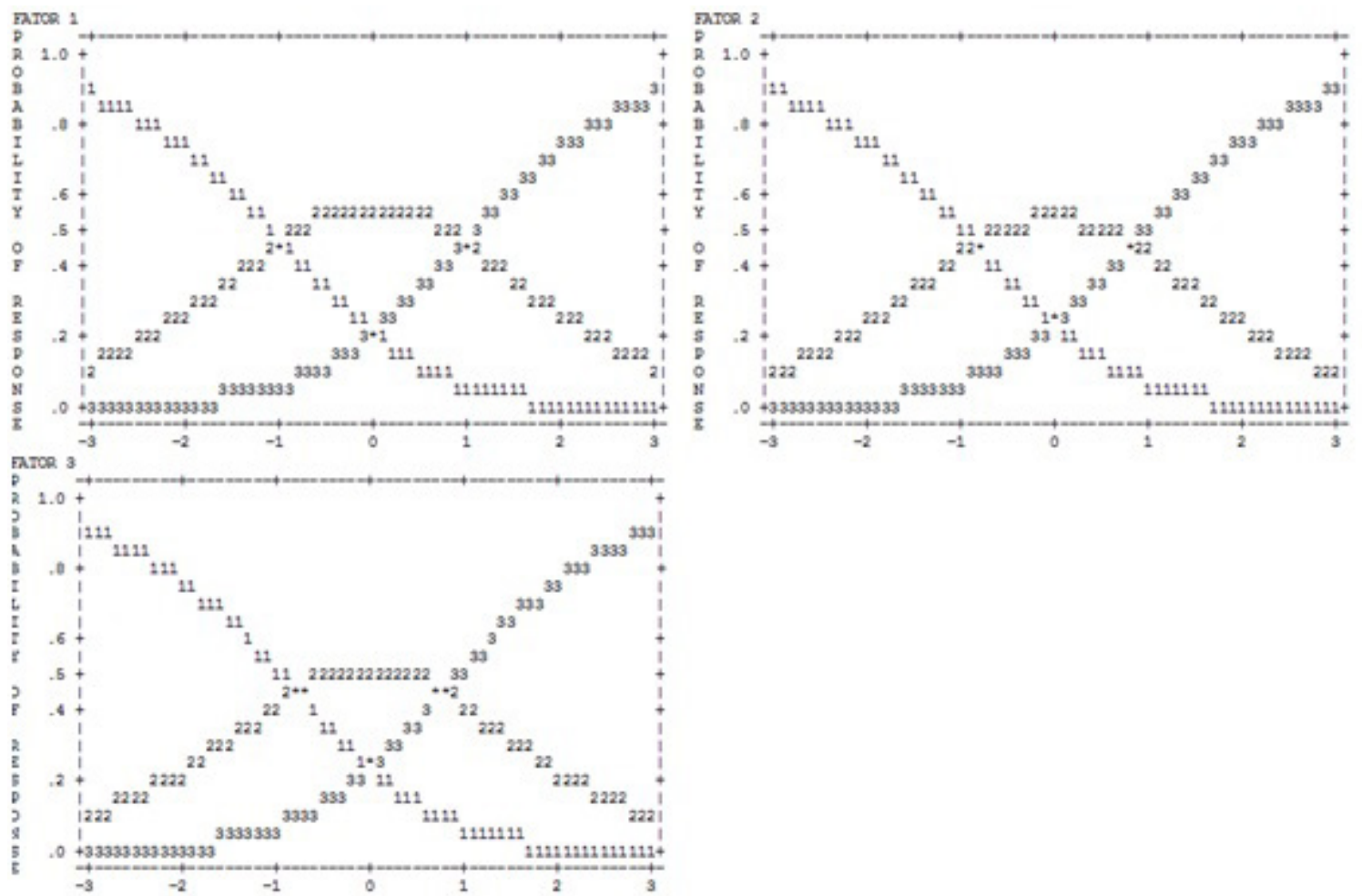

Figura 2 - Categorias de resposta do DSQ-40 após o procedimento collapsed 
Pode-se observar, por meio da Figura 2, que após o procedimento realizado, as categorias mantidas apresentaram uma clara representação, ou seja, a não sobreposição das curvas em pelo menos uma faixa de theta. Em outras palavras, a separação das curvas em diferentes regiões na escala de theta (eixo horizontal) é uma característica métrica desejável e os dados empíricos mostram que as resposta aos estímulos elaborados (itens) foi modelada quantitativamente por meio de uma relação monotônica crescente entre theta e a categoria escalar. No caso dos três fatores do instrumento, a composição que funcionou de maneira mais adequada foi a de três categorias de resposta. Ainda, cabe salientar que foi verificada uma progressão dos níveis de theta à medida que a escala Likert aumentava em todos os fatores do DSQ-40 após o procedimento de coalisão. Também é importante ressaltar que a dimensionalidade das três dimensões foi testada após a mudança na escala Likert e não foram encontradas diferenças quanto à expressão dos eigenvalues.

Na Tabela 2 estão apresentadas as estatísticas descritivas sumarizadas dos traços latentes (theta) dos respondentes, seus respectivos índices de ajuste (infit e outfit) e o número de itens respondidos em cada um dos fatores do DSQ-40. Além disso, essa tabela sumariza os dados descritivos para os itens, isto é, o nível de dificuldade, os índices de ajuste, a correlação item-theta, e os índices de fidedignidade (real e modelado, linha superior).
É possível verificar que o nível médio do traço latente para os fatores neurótico e imaturo sugerem que os itens tenderam a não serem endossados pela amostra, sendo que somente o fator maduro apresentou média positiva, sugerindo que esse foi o fator mais endossado pelos participantes. Esse dado era esperado considerando que a amostra deste estudo é composta por sujeitos da população geral e, por isso, devem apresentar características mais saudáveis e adaptativas (Blaya et al., 2004). Observou-se também variabilidade de pontuação em todos os fatores, sugerindo que a amostra é composta por pessoas com níveis de intensidade distintos em relação às características avaliadas pelo instrumento. Para realizar essa inferência, assume-se que as pontuações observadas, mais brandas ou mais extremas, são indicativas do nível de funcionamento dos sujeitos em relação aos grupos (maduro, neurótico e imaturo) de mecanismos de defesa.

Ainda em relação aos participantes, por meio dos índices de ajuste infit e outfit, foram verificadas discrepâncias entre os valores esperados e observados em relação à estimação dos thetas dos respondentes. Esses valores tenderam a ser adequados (Linacre \& Wright, 1994), uma vez que a média foi abaixo de 1,3 para todas as escalas. Entretanto, foram encontrados valores máximos dos índices de ajuste superiores ao critério utilizado, sugerindo discrepâncias para além do esperado pelo modelo para alguns sujeitos.

Tabela 2 - Estatísticas descritivas sumarizadas das pessoas e dos itens

\begin{tabular}{|c|c|c|c|c|c|c|c|c|c|}
\hline & & $\begin{array}{c}\text { Pessoas } \\
\text { Theta } \\
\end{array}$ & $\begin{array}{c}\text { Itens } \\
\text { Infit } \\
\end{array}$ & Outfit & b & Infit & Outfit & $r$ & $\alpha$ \\
\hline \multirow{3}{*}{ 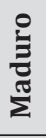 } & M (DP) & $0,05(1,00)$ & $1,02(0,49)$ & $1,02(0,50)$ & $0(0,42)$ & $1,00(0,18)$ & $1,01(0,19)$ & \multirow{3}{*}{$0,42-0,64$} & \multirow{3}{*}{$0,60(0,67)$} \\
\hline & Máx. & 2,73 & 2,56 & 2,70 & 0,58 & 1,23 & 1,70 & & \\
\hline & Mín. & $-2,50$ & 0,08 & 0,08 & $-0,70$ & 0,73 & 0,74 & & \\
\hline \multirow{3}{*}{ 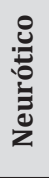 } & $\mathrm{M}(\mathrm{DP})$ & $-0,65(0,89)$ & $1,00(0,56)$ & $1,01(0,64)$ & $0(0,37)$ & $1,02(0,17)$ & $1,05(0,23)$ & \multirow{3}{*}{$0,34-0,58$} & \multirow{3}{*}{$0,18(0,31)$} \\
\hline & Máx. & 2,06 & 2,74 & 3,82 & 0,61 & 1,23 & 1,53 & & \\
\hline & Mín. & $-2,88$ & 0 & 0 & $-0,47$ & 0,82 & 0,80 & & \\
\hline \multirow{3}{*}{ 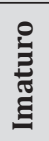 } & $\mathrm{M}$ (DP) & $-0,87(0,85)$ & $0,98(0,37)$ & $0,97(0,37)$ & $0(0,45)$ & $1,00(0,13)$ & $0,98(0,17)$ & \multirow{3}{*}{$0,19-0,61$} & \multirow{3}{*}{$0,63(0,67)$} \\
\hline & Máx. & 1,90 & 2,04 & 2,02 & 0,79 & 1,27 & 1,41 & & \\
\hline & Mín. & $-3,45$ & 0,12 & 0,13 & $-0,96$ & 0,79 & 0,68 & & \\
\hline
\end{tabular}


No que concerne aos dados descritivos para os itens, o índice de dificuldade variou entre $-0,96$ e 0,79 , que são o mínimo e o máximo do fator imaturo. A média dos índices de ajuste de todos os fatores foi adequada (inferior a 1,3), ainda que o máximo para alguns fatores tenha atingido índices superiores ao que é esperado. Ainda, as correlações item-theta apontam para moderadas e altas magnitudes de correlação entre os itens e seus respectivos fatores, o que também sugere coesão entre os componentes (itens) de cada dimensão.

Ao lado disso, o índice de fidedignidade das estimativas de theta calculado pelo modelo de Rasch variou entre 0,18 e 0,63 (real) e 0,31 e 0,67 (modelado). A ponderação acerca da adequação dos índices de fidedignidade dos conjuntos de itens deve levar em conta que ao menos dois fatores (fatores um e dois) possuem um número pequeno de itens e que o nível médio de dificuldade dos itens e o nível médio dos sujeitos no traço latente apresentam diferenças importantes (sobretudo, no fator três). Esses elementos podem influenciar no cálculo dos índices de fidedignidade (Embretson \& Reise, 2000). A ponderação acerca dessas discrepâncias pode ser levada em consideração por meio do cálculo do erro ou precisão local, que será apresentado mais adiante neste estudo.

As informações descritivas das pessoas e itens são complementadas por meio dos mapas de itens (Figura 3), a partir dos quais é possível observar que a média dos itens (representada pela letra $\mathrm{M}$ no lado direito de cada um dos mapas) se afasta de maneira crescente na escala de theta na medida em que o fator refere-se a conjuntos de mecanismos de defesas menos adaptativos. Esse dado era esperado e configura-se como evidência de validade para o instrumento, uma vez que é esperado que a população geral exiba mais frequentemente mecanismos de defesa adaptativos de defesa em relação aos mecanismos mais desajustados (Blaya et al., 2004).

Além disso, a análise visual dos mapas de itens permite verificar lacunas na mensuração do construto. Considerando os três conjuntos de itens compondo o DSQ-40, observa-se que níveis medianos e mais altos dos mecanismos de defesa estão adequadamente representados pelo instrumento; contudo, é possível observar, para os três fatores, que alguns sujeitos encontram-se em regiões inferiores na escala de theta (por exemplo, a faixa entre - 4 e -2 de theta) e não existem itens avaliando os sujeitos nessas regiões. Não foram levantados dados neste estudo que permitam explicar essa ocorrência, mas é possível levantar uma hipótese de que (a) os itens do instrumento foram formulados de maneira que alguns sujeitos não endossam itens que representam mecanismos de defesa típicos nessas pessoas e/ou (b) existem mecanismos de defesa, típicos na população, que não são avaliados pelo DSQ-40. Futuros estudos devem verificar essas possibilidades. Na continuidade, complementando as informações acerca da fidedignidade das dimensões, realizou-se também o cálculo da precisão ou erro local.

Uma das vantagens do uso da TRI é compreender a fidedignidade condicionada a escala, isto é, saber em que região da escala o teste apresenta maior índice de precisão. Isso é feito por meio da curva de informação que mostra a informação disponível em relação aos níveis de theta. Uma forma de expressar a curva em uma escala padronizada de 0 a 1 é a precisão local (Daniel, 1999).

Por meio desse índice é possível verificar para quais níveis de theta (traço latente) o conjunto de itens (fator) é mais livre de erro de medida (isto é, mais fidedigno). Assim, por exemplo, um fator com um índice moderado de fidedignidade pode ser altamente fidedigno em uma determinada faixa de traço latente, mas pouco para outra faixa. Na Figura 4 estão apresentados os índices de fidedignidade para os fatores do DSQ-40 de acordo com o nível de theta dos sujeitos (precisão local).

Na Figura 4, o eixo x (horizontal) diz respeito ao theta e o eixo y (vertical) ao índice de fidedignidade. 0 traço horizontal que corta o gráfico está dividindo a curva em índices de fidedignidade iguais ou maiores a 0,75 e índices inferiores a esse ponto de corte. A partir disso, é possível verificar em quais faixas de theta os distintos fatores do DSQ-40 são mais fidedignos. Como se vê, os fatores um (maduro) e três (imaturo) do instrumento exibem uma faixa expressiva de theta no qual a fidedignidade é igual ou superior ao ponto de corte estabelecido $(0,75)$. Em relação ao fator dois (neurótico), os índices de fidedignidade ficaram mais próximos de 0,70 com uma pequena faixa superior a esse índice. Para os três fatores, considerando faixas de theta ao redor de zero, isto é, de 1 a 2 logits acima e abaixo desse ponto, os índices de fidedignidade são expressivamente superiores aos índices gerais encontrados para os fatores, demonstrando que é nessa faixa que o instrumento 


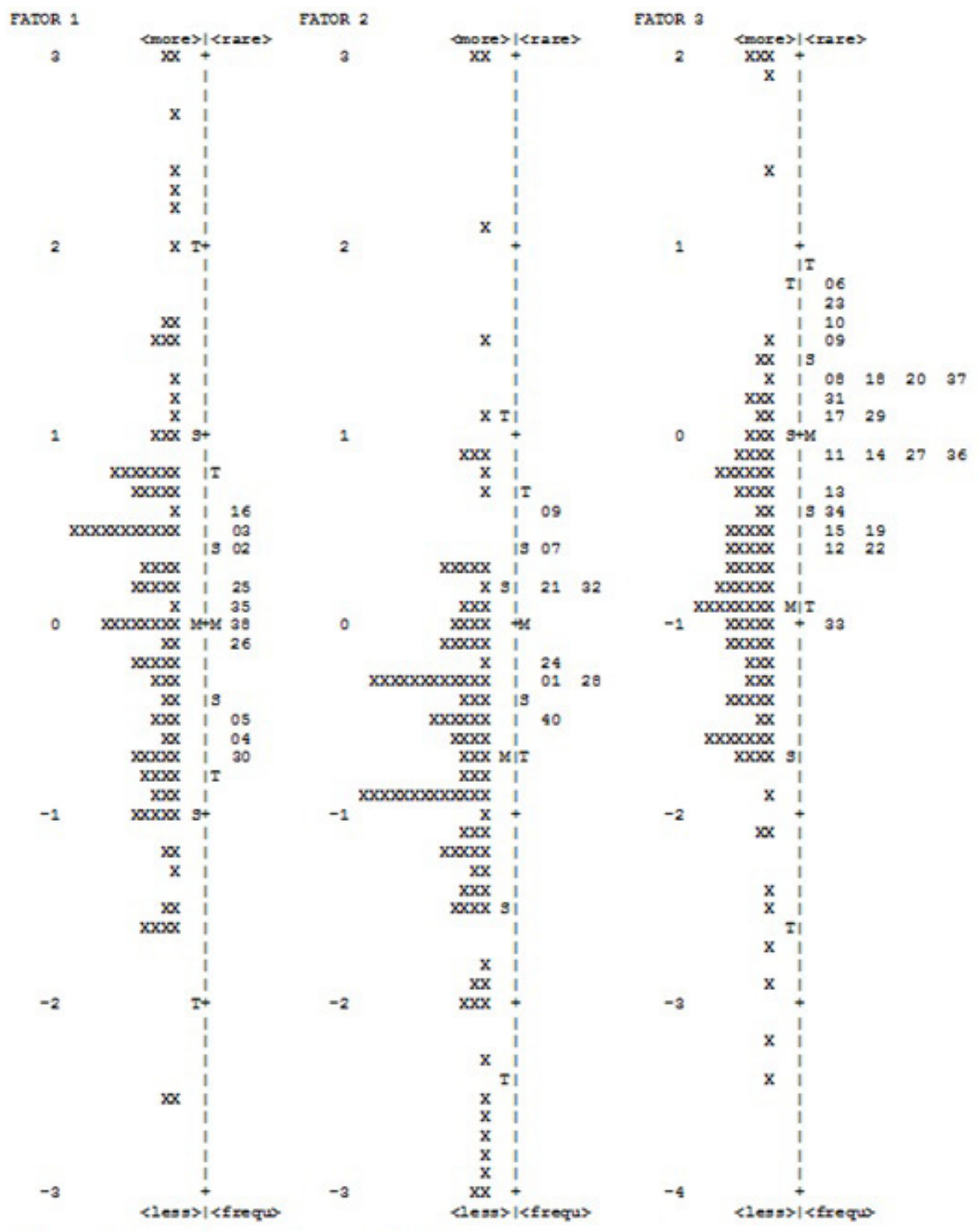

Figura 3 - Mapa de itens dos fatores do DSQ-40

avalia com uma quantidade de erro menos expressa. Verifica-se, assim, que esses dados são contrastantes com o índice "geral" de fidedignidade dos fatores, que ponderado para diferentes níveis no traço latente, tenderam a aumentar para determinadas faixas de theta.

Além disso, as faixas de theta nas quais o nível de fidedignidade foi mais alto variaram de acordo com os fatores, de modo que para o fator um a faixa abarcou níveis positivos e negativos de theta e para o fator três é possível observar uma expressão maior de thetas negativos. Isso já era esperado de acordo com a Tabela 2, considerando que o nível de fidedignidade sofre impacto de discrepâncias entre os níveis da amostra no construto latente e do conteúdo dos itens que compõem os fatores. 

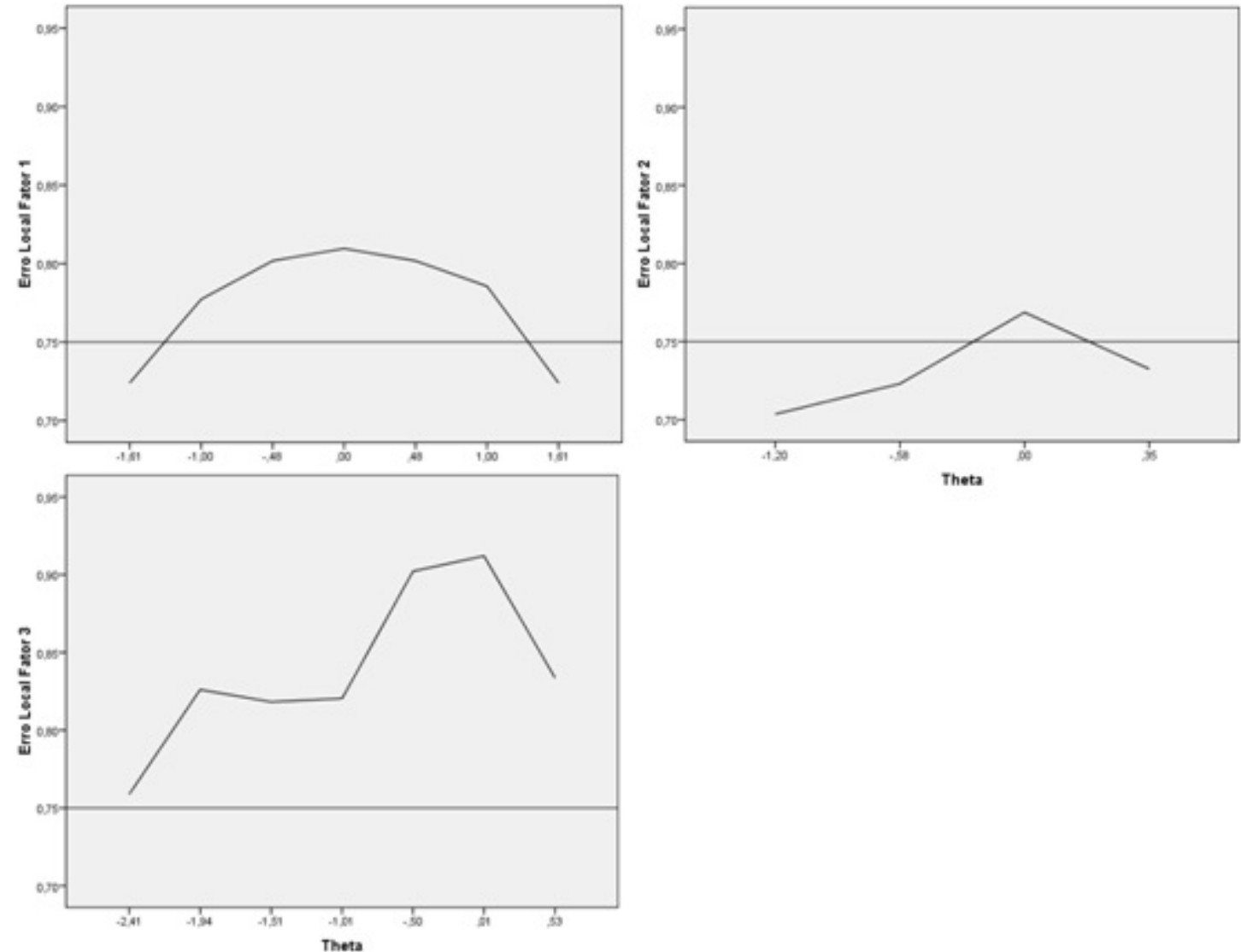

Figura 4 - Precisão local para os thetas dos fatores do DSQ-40

\section{Consideracões Finais}

Este estudo teve o objetivo de verificar os parâmetros dos itens e pessoas obtidos por meio do modelo de Rasch para os fatores do DSQ-40. De um modo geral, os dados encontrados demonstram evidências de validade para o instrumento e níveis adequados de fidedignidade.

Verificou-se que cada um dos fatores do instrumento atingiu os critérios para unidimensionalidade, o que sustenta a estrutura proposta para o instrumento. Ainda assim, o fator três apresentou possibilidade da existência de uma segunda dimensão. Ao lado disso, as categorias de resposta com base em uma escala Likert de sete pontos não foram sustentadas, e as análises sugeriram a adequação de uma escala Likert de três pontos. Futuros estudos devem investigar essa proposta.

Os níveis de theta e $b$ encontrados sugerem que os mecanismos mais adaptativos são mais comuns na população em relação aos mecanismos desadaptativos, o que era esperado para o DSQ-40. Ainda, os níveis de fidedignidade calculados pelo modelo de Rasch foram, no geral, baixos. Contudo, considerando as distintas faixas de theta, foi observado que existem níveis de theta nos quais os índices de fidediginidade tendem a ser superiores a 0.75 , o que é satisfatório do ponto de vista psicométrico.

Apesar dos dados favoráveis, devem-se considerar as limitações deste estudo, que se referem, sobretudo, acerca da amostra, que não é representativa da população em que está inserida, tendo sido composta por conveniência. Outras pesquisas devem continuar a verificação das propriedades psicométricas da versão brasileira do DSQ-40, bem como investigar a utilidade clínica da ferramenta na prática profissional.

\section{Referências}

American Psychiatric Associaton (2002). Manual diagnóstico e estatístico de transtornos mentais - DSM-IVTR (4a ed.). Porto Alegre: Artemed. 
Andrade, M. \& Shirakawa, I. (2006). Versão brasileira do Defense Style Questionnaire (DSQ) de Michael Bond: problemas e soluções. Rev. Psic. Rio Gd. Sul, Porto Alegre, 28(2), 144-160.

Andrews, G., Singh, M., \& Bond, M. (1993). The Defense Style Questionnaire. Journal of Nervous and Mental Disease , 181, 246-256.

Blaya, C., Kipper, L., Heldt, E., Isolan, L., Ceitin, L. H., Bond, M., et al. (2004). Versão em português do Defensive Style Questionnaire (DSQ - 40) para avaliação dos mecanismos de defesa: Um estudo preliminar. Revista Brasileira de Psiquiatria, 26(4), 255-258.

Blaya, C., Dorneles, M., Blaya, R., Kipper, L., Heldt, E., Isolan, L., et al. (2006). Do defense mechanisms vary according to the psychiatric disorder? Revista Brasileira de Psiquiatria, 28(3), 179-183.

Blaya, C., Dornelles, M., Blaya, R., Heldt, E., Kipper, L., Bonde, M. \& Manfro, G. G. (2007). Brazilian- Portuguese version of the Defensive Style Questionnaire (DSQ-40) for the assessment of defense mechanisms: construct validity study. Psychotherapy Research, 17, 261-72.

Daniel, M. H. (1999). Behind the scenes: using new measurement methods on the DAS and KAIT. In S. E. Embretson \& S. L. Hershberger (Eds.), The new rules of measurement: What every psychologist and educator should know (pp. 37-63). Mahwah, NJ: Lawrence Erlbaum.

Embretson S. E., \& Reise, S.P. (2000). Item response theory for psychologists. Mahwah: Lawrence Erlbaum.

Freud, S. (1976). As neuropsicoses de defesa. In: S. Freud. Obras Psicológicas Completas de Sigmund Freud. (Vol. 3, pp. 55-75). Rio de Janeiro: Imago. (Trabalho original publicado em 1894)

Freud, S. (1976). Novos comentários sobre as neuroses de defesa. In: S. Freud. Obras Psicológicas Completas de Sigmund Freud. (Vol. 3, pp. 183-200). Rio de Janeiro: Imago. (Trabalho original publicado em 1896)
Freud, A. (1937). Ego and the mechanisms of defense. London: Hogarth Press.

Galvão, L. F. (2007). Propriedades psicométricas do Defensive Style Questionnarie - 40 (DSQ-40) para a população prisional. Tese não publicada. PUC-Campinas.

Hambleton, H. K. \& Swaminatham, H. (1985). Item response theory: principles and applications. Boston: Kluwer.

Hemphill, J. F. (2003). Interpreting the magnitudes of correlation coefficients. American Psychologist, 58, 78-79.

Kipper, L., Blaya, C., Teruchkin, B., Isolan. L., Mezzono, K., Bond, M., Manfro, G. G. (2004) Evalitation of defense mechanisms in adult patients with panic disorder: before and after treatment. J. Nerv. Ment. Dis., 193(9), 619-624.

Linacre, J. M. (2009). WINSTEPS: Multiple-choice, rating scale, and partial credit Rasch analysis (Computer Software). Chicago, Illinois: MESA Press.

Pasquali, L., \& Primi, R. (2007). Fundamentos da Teoria de Resposta ao Item - TRI. Em Pasquali, L. Teoria de Resposta ao Item: Teoria, Procedimentos e Aplicações. Brasília: LabPAM/Unb.

Smith R. M. (1996) Polytomous Mean-Square Fit Statistics. Rasch Measurement Transactions, 10(3), 516-517.

Wright B. D. \& Linacre J. M. (1994). Reasonable meansquare fit values. Rasch Measurement Transactions, $8(3), 370$.

Zaslavsky, J. ; Escobar, J. (2003). Mecanismos de defesa do ego. In: A. C. Netp; G. J. C. Gauer; N. R. Furtado. (Org.). Psiquiatria para estudantes de medicina. 1a. ed. Porto Alegre: Edipucrs. v. 1, p. 40-46. 